reading ages could be entirely explained in terms of the five year history of preoperative illness. This patient's performance suggested that the right hemisphere plays no necessary role in supporting reading skills, at least of the type assessed in the study (mainly single word recognition, comprehension and promunciation). (Patterson $\mathrm{K}$ et al. Reading with one hemisphere. Brain Feb $1989 ; 112: 39-63)$.

COMMENT. Although the right hemisphere appeared to play no necessary role in supporting reading skills, perhaps it is important both for reading acquisition and for mature efficient reading. In particular, the right hemisphere may be important for quick pattern recognition during speed reading and for semantic-thematic orientation to a situation or narrative. Loss of right hemisphere contribution to reading in adults would lead to subtle deficits in higher order reading which are rarely assessed in the neurological clinic. (Zaidel and Schweiger. Cognitive Neuropsychology 1984; 1:351). The normal acquisition of reading requires two functioning hëmispheres and children with one hemisphere have difficulty learning to read whether it is the left or the right hemisphere that has been removed. The vast majority of right-handed people have left hemisphere daminance for language skills such as reading. It had been an unresolved question, however, whether the right hemisphere of a left hemisphere daminant individual also developed significant capacity for reading. Cases of hemispherectamy performed for neurological disease of late onset provide most convincing evidence regarding reading with one hemisphere.

\title{
DIET AND NEUROLOGICAL DISORDERS
}

\section{KETOGENIC DIETS IN EPILEPSY}

The classical ketogenic diet, the medium chain triglyceride (MCT) diet, and a modified MCT diet were used in the treatment of 55 children and 4 adults with intractable epilepsy at the University and Clinical Departments of Paediatrics, John Radcliffe Hospital, Oxford, England. The main types of seizures were drop attacks (24), infantile spasms (7), tonic-clonic (6), partial complex (5), primary absence (2), myoclonic absence (2), complex absence (4), and partial simple (4). Forty-five of the patients were under ten years of age. Cooperation and compliance were good and 57 patients campleted at least six weeks dietary management and same continued for periods up to four years. Fifty-six had significantly elevated blood ketones bodies and all were reported to have ketonuria. Eighty-one percent showed greater than $50 \%$ reduction in seizure frequency. The response was independent of the type of diet used; all three diets appeared to be equally effective in children under the age of 15 years. The high fat diet was found to be palatable by all of the children but the adults found the restrictions unacceptable. Large quantities of MCT oil were also found to be unpalatable by all age groups. No patient found the cream and butter content of the classical diet to be unacceptable. Analysis of variance failed to show any significant difference between the type of seizure and the success of treatment. The EEG showed improvement in 14 cases, 9 while the children were on the MCT diet and 5 while on the classical diet. Nausea, vamiting or abdominal discomfort occurred in approximately one-half the patients taking 
the MCT diet; drowsiness occurred in $25 \%$ at the introduction of the diet. Loss of ketosis occurred during the prodramal phase of an intercurrent illness and was often accompanied by an increase in the frequency of seizures. Two children under one year of age showed no increase in weight, length or head circumference during a six month period on the diet. (Schwartz R H et al. Ketogenic diets in the treatment of epilepsy: Short term clinical effects. Dev Med Child Neurol April 1989; 31:145-151).

\section{METABOLIC EFFECTS OF KETOGENIC DIETS}

The results of 24 metabolic profiles performed on 55 epileptic children receiving the classical ketogenic diet, the MCT diet, a modified MCT diet, and normal diets are reported from the University Department of Paediatrics, John Radcliffe Hospital, Oxford, England. The clinical effects of the diets are reported in the previous paper. All three therapeutic diets improved the control of epilepsy and induced a significant increase in the concentrations of blood aceto-acetate and 3-hydroxybutyrate, the greatest elevation being seen in patients on the classical diet (4:1). The pre- and post-prandial blood ketone levels with the classical diet reached a mean of $2.6 \mathrm{mmol} / \mathrm{L}$ before breakfast and $4 \mathrm{mmol} / \mathrm{L}$ before supper. The three ketogenic diets led to a buildup of ketone body concentrations during the day, reaching maximum levels in the afternoon. This was in contrast to the normal diet which led to slightly higher levels in the morning fasting samples. The Ketostix reagent strip test for urinary ketone bodies reflected these changes and showed higher levels in the afternoon and lower levels in morning samples. Children between 5 and 10 years of age showed the highest blood ketone levels. Levels of blood glycerol were highest while fasting and lowest after meals. Despite the high fat content of the diets none of the concentrations of plasma cholesterol, high density lipoproteins, low density and very low density lipoproteins was significantly raised in any of the therapeutic diet groups. Hypoglycemia was not documented in any patient at any time but blood concentrations of pyruvate were significantly lower. Lower blood levels of alanine occurred on all three diets, the most marked difference being in children receiving the classical diet. The remaining plasma amino acid concentrations tended to be lowest on the classical diet but other than alanine values, the mean concentrations of individual amino acids on the three diets failed to show any significant change. Plasma insulin concentrations corresponded to the blood glucose profiles, showing elevations after each meal, the highest levels occurring with the normal and modified MCT diet and the lowest responses occurring with the classical ketogenic diet. The mean plasma concentrations of sodium, potassium, chloride and bicarbonate did not differ significantly between the four diets, and plasma urea, creatinine, calcium, phosphate, total protein, albumin and bilirubin levels were also similar. Plasma uric acid levels were higher on all three ketogenic diets with the highest increase on the MCT diet. The mechanism of action of the ketogenic diet was not determined. (Schwartz R M et al. Metabolic effects of three ketogenic diets in the treatment of severe epilepsy. Dev Med Child Neurol April 1989; $31: 152-160)$.

COMMENT. The above two studies performed at the John Radcliffe Hospital in Oxford and including patients from the pediatric practice of Dr. B.D. Bower have reconfirmed the efficacy of the ketogenic diet in the management of intractable epilepsy in children under 15 years. The 